\section{Comparison of aversive thresholds in cats obtained by the methods of titration and constant stimuli*}

\author{
CORNELIS L. J. STOKMAN† and MURRAY GLUSMAN \\ New York State Psychiatric Institute, New York, N.Y. 10032 \\ and \\ WALLACE J. ORLOWSKY \\ Richmond College, Staten Island, N.Y. 10301
}

Aversive thresholds were measured in five cats by means of the methods of titration and constant stimuli, using two intertrial intervals (15 and $3 \mathrm{sec}$ ). The data show that at the 3-sec intertrial interval, the aversive thresholds are significantly higher than those for the $15-\mathrm{sec}$ intertrial interval only for the method of constant stimuli and not for titration. There was a significant Method by Intertrial Interval interaction effect. The results are interpreted in terms of the combined effects of conditional aversiveness and magnitude of reinforcement (duration of time-out from shock) and the differential sensitivity of the two methods to these variables.

Behavioral titration has been used extensively to measure aversive thresholds (Weiss \& Laties, 1958) and to assess the effects of drugs and brain lesions upon the perception of noxious stimulation (Ross, 1966; Kelly \& Glusman, 1968; Orlowsky \& Glusman, 1969). Aversive thresholds can be considered thresholds of "pain" only insofar as they measure the primary aversive properties of stimuli. However, an essential characteristic of the titration schedule as in the psychophysical method of limits is the sequential presentation of shock intensities in ascending order if the $S$ does not terminate or reduce the shock by a response. When low-intensity shocks, not in themselves aversive, systematically precede higher aversive shocks, the lower intensities may become conditionally aversive. In such a case, the organism may respond to the lower shocks in order to avoid the higher ones which would otherwise follow. Thus, the aversive thresholds obtained may not reflect accurately the primary aversive properties of the shock, as defined by escape behavior, but may be lowered through the avoidance behavior produced by the conditional aversive properties of weaker shocks (Boren \& Malis, 1961). Since the lower-intensity shocks acquire their aversive properties through temporal pairing with primary aversive higher shocks, the amount of conditional aversiveness in titration

* This research was supported in part by Postdoctoral Research Training Program, NIMH Grant MH-10315.

$\div$ Reprints may be obtained from Cornelis L. J. Stokman, New York State Psychiatric Institute, Department of Behavioral Physiology, 722 West $168 \mathrm{th}$ Street, New York. N.Y. 10032 . should be related to the temporal patterning of the stimuli.

Fields \& Glusman (1969) systematically varied the intertrial interval (ITI) in the titration procedure and observed that increasing the ITI resulted in a reduction of the amount of avoidance responding relative to the amount of escape responding. However, an increase in ITI in titration by separating shock levels more in time not only may reduce conditional aversiveness of the lower shocks, but also provides a longer time-out from shock for the S. The longer the time-out from shock, the greater its effectiveness as a reinforcer, i.e., longer ITIs increase the strength (decrease in latency) of an escape response to a given shock level (Dinsmoor \& Hughes, 1956; Dinsmoor, Hughes, \& Matsuoka, 1958). If response probability as an index of response strength behaves in a fashion similar to response latency, then increasing the ITI in titration increases the probability of a response to a given shock level, resulting in a lowered threshold.

A change in ITI in titration simultaneously affects both the amount of conditional aversiveness and the magnitude of reinforcement. Consequently, the aversive thresholds obtained represent the combined results of both factors operating together but in opposing fashion, i.e., a short ITI increases conditional aversiveness and lowers threshold, but at the same time it provides less reinforcement, thereby tending to increase the threshold.

The present study was designed to determine the relative influence of conditional aversiveness and magnitude of reinforcement with changes in ITI. To achieve this, titration thresholds were measured and compared with those obtained with the method of constant stimuli (CS). Since in the method of CS shock levels are presented randomly, low (nonaversive) shocks do not systematically precede higher (aversive) ones; therefore, they cannot be expected to develop conditionally aversive properties, and the thresholds obtained should reflect escape rather than a combination of escape and avoidance. The ITI affects the thresholds in the method of CS only by magnitude of reinforcement, i.e., length of time-out from shock. Comparing the thresholds obtained with the methods of $\mathrm{CS}$ and titration under different ITIs will show the relative influence of the variables of conditional aversiveness and magnitude of reinforcement.

\section{METHOD}

Five adult female cats served as Ss. They were housed in individual cages and had free access to food and milk, except during the experimental sessions.

The Ss were run in a Lehigh Valley sound-attenuated experimental chamber. Shocks, consisting of biphasic square-wave pulses of $10-\mathrm{msec}$ duration and repetition rate of $50 \mathrm{~Hz}$, were generated by a Coldspring Ins t r u m e n t $\mathrm{T}$ y e $16-\mathrm{B}$ constant-current shock source. The intensities of successive shocks could be decreased or increased in $0.3-\mathrm{mA}$ intervals over a possible range of 25 steps, or 0.0-7.2 mA. Shocks were delivered to the $\mathrm{Ss}$ via electrodes strapped to their flanks. The flanks were shaved periodically and marked with gentian violet to insure uniformity of stimulation site. A Soroban tape reader programmed the sequence of stimulus intensities and intertrial intervals.

Following initial shaping, aversive thresholds were measured with the discrete-trial titration schedule (Halpern \& Alleva, 1964). In the trial titration procedure, a series of electric shocks was presented to the cat. The intensity of the shock was under the control of the $S$. If the animal responded (pressed the lever) during the presentation of the shock, the shock was terminated immediately, and after a 15-sec ITI, the next shock was presented at a lower level. If no response was made, the shock continued for $5 \mathrm{sec}$, and after the 15 -sec ITI, the next shock was presented at a higher level. Responses during the ITI were ineffective. In this fashion, the animal established an aversive threshold by responding to some shocks but not to others. All Ss were exposed to this schedule for at least 30 sessions or until thresholds 
had stabilized as determined by visual inspection of the data. Then they were exposed to each of the four experimental conditions, i.e., CS with ITIs of 15 and $3 \mathrm{sec}$ and titration method with ITIs of 15 and $3 \mathrm{sec}$. These values were chosen because Fields \& Glusman (1969) had observed that conditional aversiveness in titration was approximately maximal at 3 -sec and minimal at 15 -sec ITIs. A counterbalanced design was chosen, with all Ss receiving all treatments. The sequence of experimental conditions was different for each cat to guard against confounding of an order effect with the experimental effect. The Ss were exposed to each condition for at least 10 sessions or until the thresholds had stabilized. Aversive thresholds, defined as the shock intensities that evoked a response $50 \%$ of the time, were determined for each session. The thresholds for the last 10 stable sessions were averaged and represent the aversive threshold under a given experimental condition. Each session consisted of 420 trials divided into three blocks of 140 trials each. In the titration procedure, the Ss controlled the range of shock intensities presented. Ss usually sampled from 5 to 10 shock levels. In the CS procedure, seven consecutive stimulus intensities were chosen because they spanned the range between $0 \%$ and $100 \%$ responding. The sequence of shock levels in the CS was randomized for a block of 140 trials. Each session consisted of three such blocks. Each shock level was presented 20 times in a given block for a total of 60 presentations per session.

An analysis of variance was performed on the data to determine the main effects of the experimental conditions and possible interactions. In addition, individual experimental conditions were compared with each other for significant differences (t test).

\section{RESULTS AND DISCUSSION}

Figure 1 shows the mean aversive thresholds obtained under the different experimental conditions. In Fig. $1 \mathrm{~A}$, the ITIs $(15+3)$ are combined and the thresholds presented are those for the different procedures (CS vs $T$ ). As can be seen in Fig. $1 \mathrm{~A}$, the thresholds obtained with the method of CS are higher than those obtained with the method of titration ( $T$ ); however, this difference is not significant $(p>.05)$. In Fig. 1B, the thresholds for the methods of constant stimuli and titration are combined $(\mathrm{CS}+\mathrm{T})$ and the differences between $3^{-}$and $15-\mathrm{sec}$ ITIs are compared for the pooled data. The thresholds for $3-\mathrm{sec}$ ITIs are significantly higher $(F=73.50$,

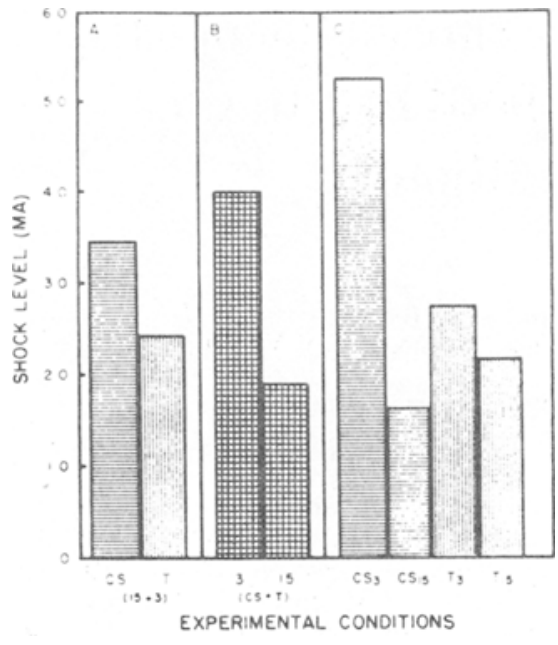

Fig. 1. Mean aversive thresholds for the different experimental conditions.

df $=1 / 4, p<.005)$ than are those for 15-sec ITIs. The thresholds for each experimental condition are shown in Fig. $1 C$, and it is clear that the 3 -sec ITI thresholds are higher than those for the 15-sec ITIs in both procedures. However, the only significant differences are those between the method of CS with 3-sec ITI $\left(\mathrm{CS}_{3}\right)$ and all other conditions $(p<.005)$. A most important finding of the present study is that the interaction between method and interval is highly significant $(\mathrm{F}=85.20, \mathrm{df}=1 / 4$, $\mathrm{p}<.001)$. Increasing the ITI from 3 to $15 \mathrm{sec}$ lowers the aversive thresholds significantly, but only for the method of CS and not for titration.

Although the counterbalancing of experimental conditions should prevent the confounding of the experimental effects with order effects, the occurrence of order effects was examined by comparing the aversive thresholds of Ss exposed to one sequence of conditions with those exposed to different sequences, e.g., the thresholds for the CS with 15-sec ITIs preceded by the CS with 3 -sec ITIs were compared with those followed by them, etc. No differences in thresholds and, therefore, no order effects were found.

The present study points to the significance of several variables affecting aversive thresholds in titration, namely, magnitude of reinforcement (length of time-out from shock), conditional aversiveness, and the interaction of these factors. These variables can be classified according to the signal detection model as nonsensory factors that influence the S's response criterion rather than the S's sensitivity, i.e., thresholds are determined not only by the stimulus parameters (e.g. intensity), but also by the consequences of the response (e.g., duration of time-out from shock). In view of the demonstrated importance of some of these criterion variables, the effects of an experimental manipulation (e.g., analgesic drug, lesion) on pain thresholds must be interpreted cautiously. It should be noted, however, that ITI or duration of time-out from shock could be described as a stimulus parameter, e.g., in terms of frequency of stimulation or repetition rate. If one considers duration of time-out from shock or ITI as a variable that affects the S's sensitivity rather than the S's response criterion, then an alternative interpretation of the data obtained in the present study is possible, i.e., in terms of adaptation rather than in terms of the effect of magnitude of reinforcement. This alternative will be discussed below. Of course, it is also possible that both the sensitivity to shock and the response criterion of the $\mathrm{S}$ may be affected by changes in ITI.

In behavioral titration, both conditional aversiveness and magnitude of reinforcement play a role, and both can be manipulated by changes in ITI, although their effects on threshold are in opposite directions. Changing the ITI from 3 to 15 sec in titration did not result ${ }^{\prime}$ in a significant change in threshold. This occurred most likely because the effectiveness of reinforcement provided by increased time-out from shock was cancelled by the reduction of conditional aversiveness. The reinforcing properties of duration of time-out from shock were demonstrated in the method of CS. Changing the ITI from 3 to $15 \mathrm{sec}$ in the CS significantly decreased the threshold. Since no sequential effects (conditional aversiveness) are present in this method, the change in threshold can be attributed to the change in magnitude of reinforcement (duration of time-out from shock).

Although the main difference between the titration and CS methods is the sequential vs random presentation of stimulus intensities, the two methods differ in other respects as well, e.g., the possible range of shock intensities. In the method of $\mathrm{CS}$, the cat always received a range of seven shock intensities. However, in the titration method, the range of shock intensities depended on the S's responding. If the animal never responded to a given intensity, but always to the next higher one, the range of shocks presented would be limited to those two. In fact, the Ss usually sampled a range between 5 and 10 intensities in the titration method. To equate approximately the range of 
intensities for the two methods, a range of seven intensities was chosen for the method of CS. Another difference between the two methods is the possible number of escape responses emitted in both methods. Although seven intensities were presented in the method of CS, the $S$ did not have to respond to all of them. In the titration method, however, the $S$ had to respond to at least six intensities if it sampled a range of seven. Inspection of the data revealed that there was no difference in total number of escape responses for the different methods or the different ITIs. Although these differences between the methods do exist, it is unlikely that they could account for the data.

Although we offer an explanation of the ITI effects on the aversive thresholds in terms of the operation of the variables of magnitude of reinforcement and conditional aversiveness, alternative explanations are possible, e.g., in terms of adaptation. It is possible that repeated exposure to shock may result in adaptation to the stimulus, i.e, it may decrease the animal's sensitivity to the shock, resulting in increased threshold. If more adaptation occurs for short ITIs than for long ITIs, one could explain the increased thresholds with
3 -sec ITIs in the method of CS in terms of a decreased sensitivity to the shock. However, Dinsmoor (1968), in reviewing the literature on adaptation to shock, concluded that insufficient evidence existed to support the existence of decreased sensitivity to shock with repeated exposure to the stimulus. Although we cannot eliminate the possibility that adaptation can totally or partly explain the data of the present study, the available evidence does not favor such an explanation.

In summary, the aversive thresholds in both the method of CS and titration can be influenced by manipulation of nonsensory variables. Both procedures are differentially sensitive to changes in ITI. At long ITIs, the two procedures result in nearly equal threshold values. At short ITIs, however, the method of titration results in significantly lower aversive thresholds than does the method of CS. We attribute this difference to the presence of avoidance responding in titration at short ITIs, which tends to produce lowering of thresholds.

\section{REFERENCES}

BOREN, J., \& MALIS, J. Determining thresholds of aversive brain stimulation. I-mprinan Journal of Physiology, 1961,
$201,429-433$

DINSMIOOR, J. A. Escape from shock as a conditioning technique. In $\mathbf{M}$. R. Jones (Ed.), Hiami symposium on the prediction of behavior, 1967: Aversice stimulation. Coral Gables, Fla: University of Miami Press, 1968.

DINSMOOR, J. A., \& HUGHES, H. L. Training rats to press a bar to turn off shock. Journal of Comparative \& Physiological Psychology, 1956, 49, $235-238$.

DINSMOOR, J. A., HUGHES, H, L., \& MATSUOKA, Y. Escape-from-shock training in a free-response situation. American Joumal of Psychology, 1958. 71, 325-337.

FIELDS L \& GLUSMAN, M. Titration of aversive thresholds in cats: Escape and avoidance components. Joumal of Comparative \& Physiological Psychology, $1969,68,334-337$.

HALPERN, L \& ALLEVA, F. Drug induced changes in thresholds for aversive stimulation in chronically implanted monkeys. Federation Proceedings, 1964, 23, Part 1, 284.

KELLY, D. D., \& GLUSMAN, M. Aversive thresholds following midbrain lesions. Journal of Comparative \& Physiological Psychology, 1968, 66, 25-34.

ORLOWSKY, W. J. \& GLUSMAN, M. Recovery of pain thresholds following midbrain lesions in the cat. Journal of Comparative \& Physiological Psychology, 1969,67, 245-251.

ROSS, G. A technique to study pain in monkeys: Effects of drugs and anatomic lesions. In R. S. Knighten and P. R. Dimke (Eds.), Pain: Henry \&., a Hospital international symposic.n. New York: Little, Brown, 1966.

WEISS, B., \& LATIES, V. Fractional escape and avoidance on a titration schedule. Science, $1958,128,1575-1576$.

\section{CURRENT LITERATURE ON ANIMAL BEHAVIOR AND PHYSIOLOGICAL PSYCHOLOGY}

Beginning with this issue, our citations of current literature are arranged in outline form so that readers may more easily find the particular articles in which they are interested. We have accumulated some backlog so that the list in this issue is longer than it will be later on. Our plan is to divide the literature on Animal and Physiological Psychology into two parts, publishing one part each month. The part covered here consists of Animal Behavior (exclusive of Animal Learning) and Physiological Psychology. The part to be published next month will consist of citations on Animal Learning. The principal headings used are given on the inside front cover along with page references.

\section{GENERAL}

LOCKARD, R. B. Reflections on the fall of comparative psychology: Is there a message for us all? American Psychologist, 1971, 26, 168-179.

\section{Genetics and Behavior}

BLIZARD, D. A. Autonomic reactivity in the rat: Effects of genetic selection for emotionality. Journal of Comparative \& Physiological Psychology, 1971, 76, 282-289.

\section{ANIMAL BEHAVIOR}

KOVACH, J. K. Interaction of innate and acquired: Color preferences and early exposure learning in chicks. Journal of Comparative \& Physiological Psychology, 1971, 75, 386-398.

ROYCE, J. R., YEUDALL, L. T., \& POLEY, W. Diallel analysis of avoidance conditioning in inbred strains of mice. Journal of Comparative \& Physiological Psychology, 1971, 76, 353-358.

SATINDER, K. P. Genotype-depen- dent effects of $d$-amphetamine sulphate and caffeine on escape-avoidance behavior of rats. Journal of Comparative \& Physiological Psychology, 1971, 76, 359-364.

SCOTT, J. P., LEE, C.-T., \& HO, J. E. Effects of fighting, genotype, and amphetamine sulfate on body temperature of mice. Journal of Comparative \& Physiological Psychology, 1971, 76, 349-352. 